\title{
THE NEUMANN PROBLEM ON LIPSCHITZ DOMAINS
}

\author{
BY DAVID S. JERISON AND CARLOS E. KENIG ${ }^{1}$
}

Let $D$ be a Lipschitz domain in $\mathbf{R}^{n}, n>2$. Let $\sigma$ denote surface measure on $\partial D$, and let $\partial / \partial n$ denote the normal derivative on $\partial D$. In this note we use an a priori estimate due to Payne and Weinberger [6], to bound the nontangential maximal function of the gradient $\nabla u$ of a (generalized) solution to the Neumann problem

$$
\Delta u=0 \quad \text { in } D ; \quad \frac{\partial}{\partial n} u=g \quad \text { on } \partial D
$$

for boundary data $g$ in $L^{2}(d \sigma)$. A corollary is that $\nabla u$ attains its boundary values nontangentially pointwise almost everywhere and through dominated convergence in $L^{2}$ on level sets that tend to $\partial D$. Moreover, $u$ belongs to the Sobolev space $H_{3 / 2}(D)$. We obtain the same bound and corollary when $u$ is the solution to the Dirichlet problem

$$
\Delta u=0 \quad \text { in } D ; \quad u=f \text { on } \partial D,
$$

where $f$ and its gradient on $\partial D$ belong to $L^{2}(d \sigma)$. For $C^{1}$ domains, these estimates were obtained by A. P. Calderón et al. [1]. For dimension 2, see (d) below.

In [4] and [5] we found an elementary integral formula (7) and used it to prove a theorem of Dahlberg (Theorem 1) on Lipschitz domains. Unknown to us, this formula had already been discovered long ago by Payne and Weinberger and applied to the Dirichlet problem in smooth domains. Moreover, they used a second formula (2), which is a variant of a formula due to F. Rellich [7], to study the Neumann problem in smooth domains. We show here that the same strategy as in [4] applied to the second formula (2) coupled with Dahlberg's theorem yields our main result. Thus integral formulas give appropriate estimates for the solution of not only the Dirichlet problem, but also the Neumann problem on Lipschitz domains. We will present a more general version that applies to variable coefficient operators, systems, and other elliptic problems in a later

\footnotetext{
Received by the editors August 18, 1980; presented to the Society at the January 1981 meeting in San Francisco.

1980 Mathematics Subject Classification. Primary 35J25, 31B20; Secondary 26B20.

$1^{1}$ The first author was supported by an NSF post-doctoral fellowship. This research was done while the first author was visiting Purdue University, whose hospitality he gratefully acknowledges. The second author was supported in part by the National Science Foundation.
} 
article. We would like to thank Professor H. F. Weinberger for calling his work to our attention.

The nontangential maximal function $M(u)$ of a function $u$ on $D$ is defined for $Q \in \partial D$ by

$$
M(u)(Q)=\sup \{|u(P)| ; P \in D,|P-Q|<2 \operatorname{dist}(P, \partial D)\} .
$$

Theorem 1 (DAhlberg [2]; SEe Also [4]). If $f \in L^{2}(d \sigma)$, then there is a unique harmonic function $u$ in $D$ such that

$$
\|M(u)\|_{L^{2}(d \sigma)} \leqslant C\|f\|_{L^{2}(d \sigma)}
$$

and $u(P) \rightarrow f(Q)$ as $P \rightarrow Q$ nontangentially for almost every $Q, d \sigma$. (The constant $C$ depends only on the Lipschitz constant of $D$.)

For simplicity we will only consider star-shaped Lipschitz domains. Let $\varphi(\theta)$ be a positive Lipschitz function on the unit sphere $S^{n-1} \subset \mathbf{R}^{n}$.

$$
D=\{(r, \theta): 0 \leqslant r<\varphi(\theta)\} .
$$

Theorem 2. Let $g \in L^{2}(d \sigma) ; \int_{\partial D} g d \sigma=0$. Then there exists a unique function $u$ such that

(a) $\Delta u=0$ in $D ; \partial u / \partial n=g$ on $\partial D$ (in the generalized sense); $\int_{\partial D} u d \sigma=0$.

$$
\|M(\nabla u)\|_{L^{2}(d \sigma)} \leqslant C\|g\|_{L^{2}(d \sigma)} .
$$

Corollary. $\partial u(P) / \partial x_{j}$ tends to a limit $\partial u(Q) / \partial x_{j} \in L^{2}(d \sigma)$ as $P \rightarrow Q$ nontangentially a.e. $Q$ and $N_{Q} \cdot \nabla u(Q)=g(Q)$, where $N_{Q}$ is the normal to $\partial D$ at $Q$.

Proof. Let $\Omega$ be a smooth domain. Denote the outer unit normal to $\partial \Omega$ at $Q$ by $N_{Q}$ and surface measure on $\partial \Omega$ by $d s$. Let $\langle$,$\rangle denote inner product.$ The normal derivative on $\partial \Omega$ is $\partial / \partial v=\left\langle N_{Q}, \nabla\right\rangle$. Denote $\alpha(Q)=Q-\left\langle Q, N_{Q}\right\rangle N_{Q}$ and define a tangential gradient by

$$
\nabla_{t}=\left(\left\langle T_{1}, \nabla\right\rangle, \ldots,\left\langle T_{n-1}, \nabla\right\rangle\right)
$$

where $N_{Q}, T_{1}, \ldots, T_{n-1}$ form an orthonormal basis at $Q$. Then $[6,3.7$ and 3.8]

$$
\begin{aligned}
& \int_{\partial \Omega}\left\{\left(|\nabla t u|^{2}-\left(\frac{\partial u}{d v}\right)^{2}\right)\left\langle Q, N_{Q}\right\rangle\right. \\
& \left.\quad-2\langle\alpha(Q), \nabla u\rangle \frac{\partial u}{\partial v}-(n-2) u \frac{\partial u}{\partial v}\right\} d s(Q)=0
\end{aligned}
$$

for any function $u$ that is harmonic in $\Omega$ and smooth in $\bar{\Omega}$. To prove this, observe that

$$
\operatorname{div}\left\{|\nabla u|^{2} Q-2\langle Q, \nabla u\rangle \nabla u-(n-2) u \nabla u\right\}=-2\langle Q, \nabla u\rangle_{:} \Delta u-(n-2) u \Delta u=0 .
$$


By Green's theorem,

$$
\int_{\partial \Omega}\left\{|\nabla u|^{2}\left\langle Q, N_{Q}\right\rangle-2\langle Q, \nabla u\rangle \frac{\partial u}{\partial v}-(n-2) u \frac{\partial u}{\partial v}\right\} d s(Q)=0 .
$$

Formula (2) follows from (3) and the fact that $|\nabla u|^{2}=|\partial u / \partial v|^{2}+\left|\nabla_{t} u\right|^{2}$.

Suppose that $\Omega$ is star-shaped: $\Omega=\{(r, \theta): 0 \leqslant r<\Psi(\theta)\}$ for some $\Psi \in C^{\infty}\left(S^{n-1}\right)$. The crucial fact is that $\left.\left\langle Q, N_{Q}\right\rangle \geqslant c\right\rangle 0$ where $c$ depends only on the Lipschitz norm of $\Psi,\|\Psi\|_{\text {Lip }}$. Therefore, we conclude from (2) that

$$
\left\|\nabla_{t} u\right\|_{L^{2}(d s)}^{2} \leqslant C\left\|\frac{\partial u}{\partial v}\right\|_{L^{2}(d s)}\left\|\left|\frac{\partial u}{\partial v}\right|+\left|\nabla_{t} u\right|+|u|\right\|_{L^{2}(d s)}
$$

where the constant $C$ depends only on $\|\Psi\|_{\text {Lip }}$. It is well known that if $\int_{\partial \Omega} u(Q) d \sigma(Q)=0$, then $\|u\|_{L^{2}(d s)}<C\left\|\nabla_{t} u\right\|_{L^{2}(d s)}$ for a constant $C$ depending only on $\|\Psi\|_{\mathrm{Lip}}$. This combined with (4) yields the a priori estimate of Payne and Weinberger $[6, \S 4]$ :

$$
\|\nabla u\|_{L^{2}(d s)} \leqslant C\left\|\frac{\partial u}{\partial v}\right\|_{L^{2}(d s)} .
$$

Applying Theorem 1 to each of the partial derivatives $\partial u / \partial x_{j}$ we find that

$$
\|M(\nabla u)\|_{L^{2}(d s)} \leqslant C\left\|\frac{\partial u}{\partial v}\right\|_{L^{2}(d s)}
$$

for a constant $C$ depending only on $\|\Psi\|_{\text {Lip }}$.

Let $\Omega_{j}=\left\{(r, \theta): 0 \leqslant r<\Psi_{j}(\theta)\right\}$ be a sequence of smooth domains containing $D$. So that $\left\|\Psi_{j}\right\|_{\text {Lip }} \leqslant 2\|\varphi\|_{\text {Lip }}, \Psi_{j} \rightarrow \varphi$ uniformly, and $\nabla_{\theta} \Psi_{j} \rightarrow \nabla_{\theta} \varphi$ pointwise almost everywhere. We will use the subscript $j$ to transfer notations from $\Omega$ to $\Omega_{j}$. The Sobolev space $H_{s}(D)$ is defined (for $s \geqslant 0$ ) as the restriction to $D$ of the Sobolev space

$$
H_{s}=\left\{h \in L^{2}\left(\mathbf{R}^{n}\right): \int|\hat{h}(\xi)|^{2}\left(1+|\xi|^{2}\right)^{s} d \xi<\infty\right\} .
$$

Let $f \in C^{\infty}\left(\mathbf{R}^{n}\right)$ satisfy $\int_{\partial D}(\partial f / \partial n) d \sigma=0$. There is a generalized solution $u \in H_{1}(D)$ to the Neumann problem (1) with $g=\partial f / \partial n$. In fact, $u=f-v$, where $v$ satisfies

$$
\int_{D}\langle\nabla v, \nabla \psi\rangle=\int_{D} \Delta f \psi \quad \text { for all } \psi \in H_{1}(D) .
$$

To specify $u$ uniquely we impose the condition $\int_{\partial D} u d \sigma=0$. Let $h(P)=|P|^{2}$. Note that $\int_{\partial \Omega_{j}}\left(\partial h / \partial v_{j}\right) d s_{j}=2 n \operatorname{vol}\left(\Omega_{j}\right) \neq 0$. Thus, there is a sequence $c_{j} \rightarrow 0$ such that

$$
\int_{\partial \Omega_{i}} \frac{\partial}{\partial v_{i}}\left(f+c_{j} h\right) d s_{j}=0
$$


Therefore, we can solve the Neumann problem

$$
\int_{\Omega_{j}}\left\langle\nabla v_{j}, \nabla \psi\right\rangle=\int_{\Omega_{j}} \Delta\left(f+c_{j} h\right) \psi \quad \text { for } \psi \in H_{1}\left(\Omega_{j}\right) \text { with } u_{j}=f+c_{j} h-v_{j}
$$

and $\int_{\partial \Omega_{j}} u_{j} d s_{j}=0$. Estimate (5) tells us that for large $j$

$$
\left\|M\left(\nabla u_{j}\right)\right\|_{L^{2}\left(d s_{j}\right)} \leqslant C\left\|\frac{\partial}{\partial v_{j}}\left(f+c_{j} h\right)\right\|_{L^{2}\left(d s_{j}\right)} \leqslant 2 C\left\|\frac{\partial f}{\partial n}\right\|_{L^{2}(d s)} .
$$

In particular, $\left\|u_{j}\right\|_{H_{1}\left(\Omega_{j}\right)}$ is uniformly bounded. Therefore, replacing $u_{j}$ by a subsequence, we can assume that $u_{j}$ converges weakly in $H_{1}(D)$ to a function $\tilde{u}$. Furthermore, for $\psi \in H_{1}$, letting $\tilde{v}=f-\tilde{u}$,

$$
\begin{aligned}
\int_{D}\langle\nabla \tilde{v}, \nabla \psi\rangle & =\lim _{j \rightarrow \infty} \int_{D}\left\langle\nabla v_{j}, \nabla \psi\right\rangle \\
& =\lim _{j \rightarrow \infty} \int_{\Omega_{j}} \Delta\left(f+c_{j} h\right) \psi-\int_{\Omega_{j} \backslash D}\left\langle\nabla v_{j}, \nabla \psi\right\rangle=\int_{D} \Delta f \psi
\end{aligned}
$$

since $\left|\int_{\Omega_{j} \backslash D}\left\langle\nabla v_{j}, \nabla \psi\right\rangle\right| \leqslant\left(\int_{\Omega_{j}}\left|\nabla v_{j}\right|^{2}\right)^{1 / 2}\left(\int_{\Omega_{j} \backslash D}|\nabla \psi|^{2}\right)^{1 / 2}$ tends to zero as $j \rightarrow \infty$. It is easy to deduce from $\int_{\partial \Omega_{j}} u_{j} d s_{j}=0$ that $\int_{\partial D} \tilde{u} d \sigma=0$. In all, $\tilde{u}=u$. Because the functions $u_{j}$ are harmonic, $\nabla u_{j} \rightarrow \nabla u$ uniformly on compact subsets of $D$. Therefore, using (6), $\|M(\nabla u)\|_{L^{2}(d \sigma)} \leqslant C\|\partial f / \partial n\|_{L^{2}(d \sigma)}$. The collection of functions $\partial f / \partial n$ is clearly dense in the space of $g \in L^{2}(d \sigma)$ with $\int_{\partial D} g d \sigma=0$ and Theorem 2 follows.

TheORem 3. Let $u$ be the solution to the Dirichlet problem $\Delta u=0$ on $D$, $u=f$ on $\partial D$ for $f \in L^{2}(d \sigma)$. If $\nabla_{t} f \in L^{2}(d \sigma)$, then

$$
\|M(\nabla u)\|_{L^{2}(d \sigma)} \leqslant C\left\|\nabla_{t} f\right\|_{L^{2}(d \sigma)} .
$$

Proof. The proof is similar to that of Theorem 2, with the rôles of $\partial u / \partial v$ and $\nabla_{t} u$ reversed.

REMARKS. (a) Let $G$ be the Green function for $D$ with pole at 0 . Apply (2) or (3) to $G$ in the domain $\Omega \backslash B_{\epsilon}\left(B_{\epsilon}\right.$ is a ball around 0 of radius $\epsilon$ ). Letting $\epsilon \rightarrow 0$ we obtain

$$
\int_{\partial \Omega}\left\langle Q, N_{Q}\right\rangle \frac{\partial G}{\partial v}(Q)^{2} d s(Q)=\frac{1}{\omega_{n}} \int_{\partial \Omega}|Q|^{2-n} \frac{\partial G}{\partial v}(Q) d s(Q) .
$$

(See [6, 5.6] and [4] for shorter proofs.)

(b) The area integral estimate [3] says that a solution $u$ from Theorem 2 or 3 satisfies $\int_{D}\left|\nabla^{2} u(X)\right|^{2} \operatorname{dist}(X, \partial D) d X<\infty$. It follows from real interpolation that $u \in H_{3 / 2}(D)$.

(c) The estimates obtained here show that (2) and (3) are actually valid in Lipschitz domains for functions $u$ satisfying Theorem 2 or 3. Moreover, (7) is valid on Lipschitz domains.

(d) The analogous estimates for the Neumann problem in dimension two were proved by Fabes and Kenig. They showed that for each Lipschitz domain 
$D \subset \mathbf{R}^{2}$, there is $p_{0}>2$ such that if $p<p_{0}, g \in L^{p}(d \sigma)$ and $\Delta u=0$ on $D$, $\partial u / \partial n=g$ on $\partial D$, then $\|M(\nabla u)\|_{L} p_{(d \sigma)} \leqslant C\|g\|_{L} p_{(d \sigma)}$. For $p \geqslant p_{0}$, the estimate fails. Also, given $p>2$, there exists a Lipschitz domain $D$ for which the estimate fails. The situation for $g \in L^{p}(d \sigma), p<2$, in higher dimensions remains open.

\section{REFERENCES}

1. A. P. Calderón, C. P. Calderón, E. Fabes, M. Jodeit, and N. M. Rivière, Applications of the Cauchy integral on Lipschitz curves, Bull. Amer. Math. Soc. 84 (1978), 287-290.

2. B. E. J. Dahlberg, Estimates of harmonic measure, Arch. Rat. Mech. Anal. 65 (1977), 275-288.

3. - Weighted norm inequalities for the Lusin area integral and the non-tangential maximal functions for functions harmonic in a Lipschitz domain, Studia Math. (to appear).

4. D. S. Jerison and C. E. Kenig, An identity with applications to harmonic measure, Bull. Amer. Math. Soc. (N. S.) 2 (1980), 447-451.

5. - The Dirichlet problem in non-smooth domains, Ann. of Math. (to appear).

6. L. E. Payne and H. F. Weinberger, New bounds in harmonic and biharmonic problems, J. Math. Phys. 33 (1954), 291-307.

7. F. Rellich, Darstellung der Eigen werte von $\Delta u+\lambda u$ durch ein Randintegral, Math. Z. 46 (1940), 635-646.

DEPARTMENT OF MATHEMATICS, UNIVERSITY OF CHICAGO, CHICAGO, ILLINOIS 60637

SCHOOL OF MATHEMATICS, UNIVERSITY OF MINNESOTA, MINNEAPOLIS, MINNESOTA $\mathbf{5 5 4 5 5}$ 